\title{
Perfil de mortalidade hospitalar por causas externas no Estado de Pernambuco: um estudo epidemiológico
}

\author{
Hospital mortality profile due to external causes in the State of Pernambuco: an epidemiological \\ study \\ Perfil de la mortalidad hospitalaria por causas externas en el Estado de Pernambuco: un estudio \\ epidemiológico
}

\author{
Germana Maria dos Santos \\ ORCID: https://orcid.org/0000-0001-5726-7900 \\ Faculdade Maurício de Nassau, Brasil \\ E-mail: germanass369@gmail.com \\ Manoel Felipe Nunes da Rocha \\ ORCID: https://orcid.org/0000-0001-5325-888X \\ Faculdade Maurício de Nassau, Brasil \\ E-mail: manoelfelipeenfermeiro@gmail.com \\ Monalis Fernanda Soares Silva \\ ORCID: https://orcid.org/0000-0002-6795-2829 \\ Faculdade Maurício de Nassau, Brasil \\ E-mail: monalisfernanda@gmail.com \\ Juliana Temóteo da Silva \\ ORCID: https://orcid.org/0000-0002-1365-6098 \\ Faculdade Maurício de Nassau, Brasil \\ E-mail: silvajulianat525@gmail.com
}

\begin{abstract}
Resumo
Objetivo: Descrever e analisar a mortalidade hospitalar por causas externas em vítimas maiores de 1 ano, no estado de Pernambuco, entre 2015 e 2019. Metodologia: Estudo epidemiológico, descritivo de série histórica, com base nos casos de óbitos notificados ao SIH / SUS do o estado de Pernambuco; com um corte de 5 anos (2015 a 2019). Foram analisadas as seguintes variáveis: grande grupo de causas, categorias de causas, faixa etária, sexo e grupo de causas. Resultados: O número de óbitos no período de 2015 a 2019 incluiu 269651 casos, sendo 72,80\% homens e 27,20\% mulheres. O maior número de casos notificados foi observado em indivíduos entre 20 e 29 anos, representando $18,25 \%$ do total. Em relação à incidência de óbitos entre os sexos, houve um crescimento considerável em 2017 , passando de 55,19 / 1000 para 59,43 / 1000 habitantes. A causa de morte mais prevalente em todas as faixas etárias foi a exposição a outros fatores específicos, com maior incidência afetando a faixa etária entre 20 e 59 anos, representando $56,75 \%$ do total de óbitos nessa faixa etária. Conclusão: Conforme apontado acima, há necessidade de atualização do SIH, melhorando as notificações na rede privada de atenção hospitalar, resultando em um número mais confiável na amostra coletada e evitando também o aumento da subnotificação.
\end{abstract}

Palavras-chave: Causas externas; Registros de mortalidade; Perfil de saúde.

\begin{abstract}
Objective: To describe and analyze hospital mortality from external causes in victims older than 1 year, in the state of Pernambuco, between 2015 and 2019. Methodology: Epidemiological study, descriptive of historical series, based on cases of deaths reported to the SIH / SUS of the the state of Pernambuco; with a 5-year cut-off (2015 to 2019). The following variables were analyzed: large group of causes, categories of causes, age group, sex and group of causes. Results: The number of deaths from 2015 to 2019 included 269,651 cases, $72.80 \%$ men and $27.20 \%$ women. The largest number of reported cases was observed in individuals between 20 and 29 years old, representing $18.25 \%$ of the total. Regarding the incidence of deaths between the sexes, there was a considerable increase in 2017, from 55.19 / 1000 to 59.43 / 1000 inhabitants. The most prevalent cause of death in all age groups was exposure to other specific factors, with a higher incidence affecting the age group between 20 and 59 years, representing $56.75 \%$ of all deaths in this age group. Conclusion: As pointed out above, there is a need to update the SIH, improving notifications in the private hospital care network, resulting in a more reliable number in the collected sample and also avoiding an increase in underreporting.
\end{abstract}

Keywords: External causes; Mortality records; Health profile. 


\begin{abstract}
Resumen
Objetivo: Describir y analizar la mortalidad hospitalaria por causas externas entre víctimas mayores de 1 año, en el estado de Pernambuco, entre 2015 y 2019. Metodología: Estudio epidemiológico, descriptivo de series históricas, basado en casos de muerte notificados al SIH / SUS del estado de Pernambuco; con un corte de tiempo de 5 años (2015 a 2019). Se analizaron las siguientes variables: gran grupo de causas, categorías de causas, grupo de edad, sexo y grupo de causas. Resultados: El número de defunciones en el período de 2015 a 2019 incluyó 269651 casos, de los cuales el $72,80 \%$ fueron hombres y el $27,20 \%$ mujeres. El mayor número de casos notificados se observó en individuos entre 20 y 29 años, representando el 18,25\% del total. En cuanto a la incidencia de defunciones entre sexos, hubo un crecimiento considerable en 2017, pasando de 55,19 / 1000 a 59,43 / 1000 habitantes. La causa de muerte más prevalente en todos los grupos de edad fue la exposición a otros factores específicos, afectando con mayor incidencia al grupo de edad entre 20 y 59 años, representando el 56,75\% del total de muertes en este grupo de edad. Conclusión: Como se indicó anteriormente, existe la necesidad de una actualización en el SIH, mejorando las notificaciones en la red de atención hospitalaria privada, dando como resultado un número más confiable en la muestra recolectada y también evitando un aumento en el subregistro.
\end{abstract}

Palabras clave: Causas externas; Registros de mortalidad; Perfil de salud.

\title{
1. Introdução
}

O adoecimento e mortalidade populacional, no Brasil, sofreu uma grande mudança em seu perfil, nos últimos tempos, devido as transições demográficas, tecnológicas sociais e econômicas que ocorreram em todo mundo. De maneira que as altas taxas de mortalidade por doenças infectocontagiosas deu lugar as relacionadas as doenças crônicas não transmissíveis (DCNT) e as causas externas (Batista, Barreto, Merino, Fracasso, Baldissera, 2018; Oliveira, Correa, Silva, Mozer, Medeiros, 2017; Messias, Bandeira, Lopes, Silva, Curado, 2018).

As causas externas são fenômenos provenientes do meio externo que através de um efeito, seja ele de gradiente de força, químico ou radioativo, são capazes de gerar dano ao organismo humano, de maneira que tais lesões podem desencadear uma série de efeitos indesejáveis, como incapacidade física e neurológica; ou até mesmo, dependendo do dano, pode desencadear a morte do indivíduo, se configurando assim como um problema sério de saúde pública (Messias, Bandeira, Lopes, Silva, Curado, 2018; Boone, Costa, Tana, Lopes, Cortes, Freitas, Alves, 2018; Cruz,2017).

Constituem-se assim as mortes por causas externas as provenientes de acidentes de trânsito, de quedas, lesões autoprovocadas (suicídio) ou provocadas por outras pessoas (homicídio), as provenientes de envenenamento, queimaduras, afogamento, acidentes de trabalho, ou até mesmo as relacionadas a abuso sexual, físicos e psicológico. Sendo que isso faz com que as causas externas sejam consideradas uma das principais causas de morte e incapacidade física em todo o mundo (Boone, Costa, Tana, Lopes, Cortes, Freitas, Alves, 2018; Fuck, Assis, Tornquist, 2020).

Responsável por mais de 5 milhões de mortes a nível mundial e 12,5 a 12,9 das mortes no Brasil, as causas externas se caracterizaram na última década como sendo a primeira ou terceira causa de morte populacional, atingindo em especial homens e pessoas com níveis socioeconômicos mais baixos, isso devido aos aspectos sociais, políticos e econômicos que influenciaram diretamente em uma urbanização desorganizada e marginalização das pessoas mais desfavorecidas (Batista, Barreto, Merino, Fracasso, Baldissera, 2018; Lignani, Villela, 2013; Messias, Bandeira, Lopes, Silva, Curado, 2018).

Levando em consideração as taxas de mortalidade, relacionadas às causas externas, a literatura demonstra que para possíveis reduções dessas é necessário que se tenha um entendimento do problema de maneira completa, para isso é de suma importância o agrupamento, análise e questionamentos acerca das lesões ocasionadas por traumas e outros fatores que se constituíram como fatais ou não, de maneira que a partir destes dados é possível identificar o perfil clínico e epidemiológico das lesões e dos problemas de saúde da população (Lignani, Villela, 2013; Messias, 2016).

Desse modo, é importante ressaltar que no Brasil o sistema responsável por registro disponibilização dos dados de mortalidade é o Sistema de Informação sobre Mortalidade (SIM), do Ministério da Saúde (MS), instituído na década de 70, o mesmo se apresenta como importante instrumento para subsidiar pesquisas a respeitos estatísticas de mortalidade e características tanto epidemiológicas quanto sociais, fornecendo assim contribuições no desenvolvimento de políticas públicas 
e ações de saúde (Messias, 2016; Rodrigues, Arruda, 2020).

Visto isso, o estudo tem como principal objetivo descrever e analisar a mortalidade hospitalar por causas externas entre vítimas acima de 1 ano de idade, no estado de Pernambuco, no período de 2015 a 2019.

\section{Metodologia}

Estudo epidemiológico, descritivo de série histórica, realizado no Estado de Pernambuco, com dados obtidos a partir do Sistema de Informações Hospitalares do Sistema Único de Saúde (SIH/SUS), disponibilizados pelo Departamento de Informática do Sistema Único de Saúde. A escolha do período 2015 a 2019 ocorreu pela disponibilidade desses dados no DATASUS no momento da coleta, em julho de 2020.

Foi dado prioridade por trabalhar somente com os casos notificados caracterizados pelos óbitos hospitalares decorrentes de causas externas ao invés de agregar, também, os casos de internação provenientes das mesmas causas, o que demandaria a necessidade de recorrer à Secretaria estadual para a coleta de tais dados, inviabilizando a proposta da pesquisa de utilizar como fonte de coleta o Sistema de Informação em Saúde (DATASUS, 2020).

O estudo foi realizado com base nos casos de óbito notificados ao SIH/SUS do estado de Pernambuco. Foram analisadas as seguintes variáveis de preenchimento obrigatório do Sistema de Informações Hospitalares: grande grupo de causas, categorias de causas, faixa etária, sexo e grupo de causas.

Pernambuco possui uma extensão territorial de $98.076,0 \mathrm{Km}^{2}$ e registrou uma população estimada, no ano de 2019, de 9.557.071 habitantes. O estado possui como capital Recife (IBGE, 2019).

Os dados foram apresentados em três tabelas e uma figura de distribuição de frequências e de coeficientes de incidência de mortalidade para cada 100.000 habitantes, construídos com o auxílio do software Excel para Windows Vista e do Programa TabNet no Ministério da Saúde. Os coeficientes de incidência definem-se como medidas por excelência do risco da doença e do agravo. Os valores calculados seguiram como base as fórmulas recomendadas para estudos epidemiológicos. Para o cálculo de incidência anual dos óbitos, foram usadas como denominadores as populações dos censos demográficos de 2015 a 2019, fornecidos Departamento de Informática do Sistema Único de Saúde (DATASUS, 2020; Melo, Pimenta, Donalisio, 2016).

\section{Resultados e Discussão}

O número de óbitos no período de 2015 a 2019, em todas as faixas etárias no estado de Pernambuco, incluiu 269.651 casos, dos quais $184.212(72,80 \%)$ eram homens e 83.691 (27,20\%) eram mulheres, caracterizando uma razão de masculinidade para o $\mathrm{n}$ total de 26,8:1.

O maior número de casos notificados, em todo o período estudado, foi observado em indivíduos entre 20 a 29 anos de idade $(\mathrm{n}=49.223)$ representando $18,25 \%$ do total (Tabela 1$)$. 
Tabela 1 - Proporção de óbitos por sexo segundo variável faixa etária e razão de masculinidade. Pernambuco, 2015 a 2019.

\begin{tabular}{|c|c|c|c|c|c|c|c|}
\hline \multirow{2}{*}{ Faixa etária } & \multicolumn{2}{|c|}{ Masculino } & \multicolumn{2}{|c|}{ Feminino } & \multicolumn{2}{|c|}{ Total } & \multirow{2}{*}{ Razão de masculinidade } \\
\hline & $\mathbf{N}^{\mathbf{0}}$ & $\%$ & $\mathbf{N}^{\mathbf{0}}$ & $\%$ & $\mathbf{N}^{\mathbf{0}}$ & $\%$ & \\
\hline 1 a 4 anos & 6067 & 59,33 & 4159 & 40,67 & 10226 & 3,79 & $1,5: 1$ \\
\hline 5 a 9 anos & 8382 & 67,47 & 4041 & 32,53 & 12423 & 4,61 & $2,1: 1$ \\
\hline 10 a 14 anos & 10839 & 78,83 & 2910 & 21,17 & 13749 & 5,10 & $3,7: 1$ \\
\hline 15 a 19 anos & 16029 & 82,17 & 3479 & 17,83 & 19508 & 7,23 & $4,6: 1$ \\
\hline 20 a 29 anos & 40558 & 82,40 & 8665 & 17,60 & 49223 & 18,25 & $4,7: 1$ \\
\hline 30 a 39 anos & 35167 & 78,53 & 9615 & 21,47 & 44782 & 16,61 & $3,7: 1$ \\
\hline 40 a 49 anos & 27065 & 73,93 & 9543 & 26,07 & 36608 & 13,58 & $2,8: 1$ \\
\hline 50 a 59 anos & 18509 & 63,16 & 10794 & 36,84 & 29303 & 10,87 & $1,7: 1$ \\
\hline 60 a 69 anos & 11048 & 50,60 & 10788 & 49,40 & 21836 & 8,10 & $1,0: 1$ \\
\hline 70 a 79 anos & 6435 & 38,37 & 10334 & 61,63 & 16769 & 6,22 & $0,6: 1$ \\
\hline 80 anos e mais & 4113 & 30,52 & 9363 & 69,48 & 13476 & 5,00 & $0,4: 1$ \\
\hline Total & 184212 & 100 & 83691 & 100 & 269651 & 100 & $26,8: 1$ \\
\hline
\end{tabular}

Fonte: Ministério da Saúde - Sistema de Informações Hospitalares do SUS (SIH/SUS).

Em relação ao tipo de causa entre os sexos, a maior porcentagem ocorreu outras causas externas de lesões acidentais representando 38,22\% ( $\mathrm{n}=188.816)$ do total de óbitos, sendo que 127.356 ocorreram em homens e 61.460 entre as mulheres caracterizando uma razão de masculinidade de 2,1:1, seguida de exposição acidental a outros fatores e aos não especificados, representando $27,55 \%(\mathrm{n}=136.110)$, sendo 97.374 casos no sexo masculino $(71,54 \%)$ e 38736 casos no sexo feminino $(28,46 \%)$ (Tabela 2$)$. 
Tabela 2 - Proporção de óbitos por causas externas segundo sexo, tipo de causa e razão de masculinidade. Pernambuco, 2015 a 2019 .

\begin{tabular}{|c|c|c|c|c|c|c|c|}
\hline \multirow{2}{*}{ Tipos de causa } & \multicolumn{2}{|c|}{ Masculino } & \multicolumn{2}{|c|}{ Feminino } & \multicolumn{2}{|c|}{ Total } & \multirow{2}{*}{$\begin{array}{c}\text { Razão de } \\
\text { masculinidade }\end{array}$} \\
\hline & $\mathbf{N}^{\mathbf{0}}$ & $\%$ & $\mathbf{N}^{\mathbf{0}}$ & $\%$ & $\mathbf{N}^{\mathbf{0}}$ & $\%$ & \\
\hline Acidentes de transporte & 27858 & 79,78 & 7062 & 20,22 & 34920 & 7,07 & $3,9: 1$ \\
\hline Pedestre traumatizado acid transporte & 6364 & 68,28 & 2957 & 31,72 & 9321 & 1,89 & $2,2: 1$ \\
\hline Ciclista traumatizado acid transporte & 2337 & 80,31 & 573 & 19,69 & 2910 & 0,59 & $4,1: 1$ \\
\hline Motociclista traumatizado acid transp & 15867 & 85,75 & 2637 & 14,25 & 18504 & 3,75 & $6,0: 1$ \\
\hline Outros acid transporte terrestre & 1345 & 83,33 & 269 & 16,67 & 1614 & 0,33 & $5,0: 1$ \\
\hline Outros acid transporte e os não especific & 1054 & 79,61 & 270 & 20,39 & 1324 & 0,27 & $3,9: 1$ \\
\hline Outras causas externas de lesões acident & 127356 & 67,45 & 61460 & 32,55 & 188816 & 38,22 & $2,1: 1$ \\
\hline Quedas & 21566 & 52,42 & 19576 & 47,58 & 41142 & 8,33 & $1,1: 1$ \\
\hline Acidentes de transporte & 27858 & 79,78 & 7062 & 20,22 & 34920 & 7,07 & $3,9: 1$ \\
\hline Pedestre traumatizado acid transporte & 6364 & 68,28 & 2957 & 31,72 & 9321 & 1,89 & $2,2: 1$ \\
\hline Ciclista traumatizado acid transporte & 2337 & 80,31 & 573 & 19,69 & 2910 & 0,59 & $4,1: 1$ \\
\hline Motociclista traumatizado acid transp & 15867 & 85,75 & 2637 & 14,25 & 18504 & 3,75 & $6,0: 1$ \\
\hline Outros acid transporte terrestre & 1345 & 83,33 & 269 & 16,67 & 1614 & 0,33 & $5,0: 1$ \\
\hline Outros acid transporte e os não especific & 1054 & 79,61 & 270 & 20,39 & 1324 & 0,27 & $3,9: 1$ \\
\hline Outras causas externas de lesões acident & 127356 & 67,45 & 61460 & 32,55 & 188816 & 38,22 & $2,1: 1$ \\
\hline Exposição a forças mecânicas inanimadas & 3022 & 81,41 & 690 & 18,59 & 3712 & 0,75 & $4,4: 1$ \\
\hline Expos cor.elétr,rad.,temper pressão extrem & 2058 & 66,86 & 1020 & 33,14 & 3078 & 0,62 & $2,0: 1$ \\
\hline Exposição às forças da natureza & 1322 & 64,27 & 735 & 35,73 & 2057 & 0,42 & $1,8: 1$ \\
\hline Expos acid a outr fatores e não especific & 97374 & 71,54 & 38736 & 28,46 & 136110 & 27,55 & $2,5: 1$ \\
\hline Agressões & 5390 & 87,36 & 780 & 12,64 & 6170 & 1,25 & $6,9: 1$ \\
\hline Eventos cuja intenção é indeterminada & 11935 & 63,80 & 6772 & 36,20 & 18707 & 3,79 & $1,8: 1$ \\
\hline Complic assistência médica e cirúrgica & 5066 & 59,68 & 3422 & 40,32 & 8488 & 1,72 & $1,5: 1$ \\
\hline Reaç anorm ou compl tard ou outros proced & 3225 & 58,17 & 2319 & 41,83 & 5544 & 1,12 & $1,4: 1$ \\
\hline Seqüelas de causas externas & 5328 & 60,86 & 3426 & 39,14 & 8754 & 1,77 & $1,6: 1$ \\
\hline Fatores suplement relac outras causas & 1740 & 60,86 & 1119 & 39,14 & 2859 & 0,58 & $1,6: 1$ \\
\hline Total & 340207 & 100 & 153823 & 100 & 494030 & 100 & $53,6: 1$ \\
\hline
\end{tabular}

Fonte: Ministério da Saúde - Sistema de Informações Hospitalares do SUS (SIH/SUS).

Para uma melhor compreensão do estudo, foi calculada a incidência de óbitos entre os sexos. Observou-se que há um crescimento considerável, em 2017, passando de 55,19/1000 para 59,43/1000 habitantes. Entre o sexo masculino houve um grande aumento, em 2017, (84,33 habitantes) no qual ocorreu sua maior incidência. Em relação ao sexo feminino, o aumento se dá no ano de 2019 (37,34/1000 habitantes).

Dessa maneira, foi possível observar que a incidência de óbitos masculinos apresentou uma queda entre 2017 e 2018 (84,33/1000 para 81,05/1000 habitantes), contudo, quando comparado aos anos de 2015 a 2019 observou-se um crescimento acentuado nas taxas. Para o sexo feminino não foi diferente, houve uma queda entre os anos de 2017 e 2018 (36,07/1000 para 35,06/1000 habitantes), porém, quando comparado aos anos de 2015 a 2019 observou-se um crescimento significativos nas taxas (Figura 1). 
Figura 1 - Coeficiente de incidência de óbitos (por 1000 habitantes), segundo o ano de notificação e sexo. Pernambuco, 2015 a 2019.

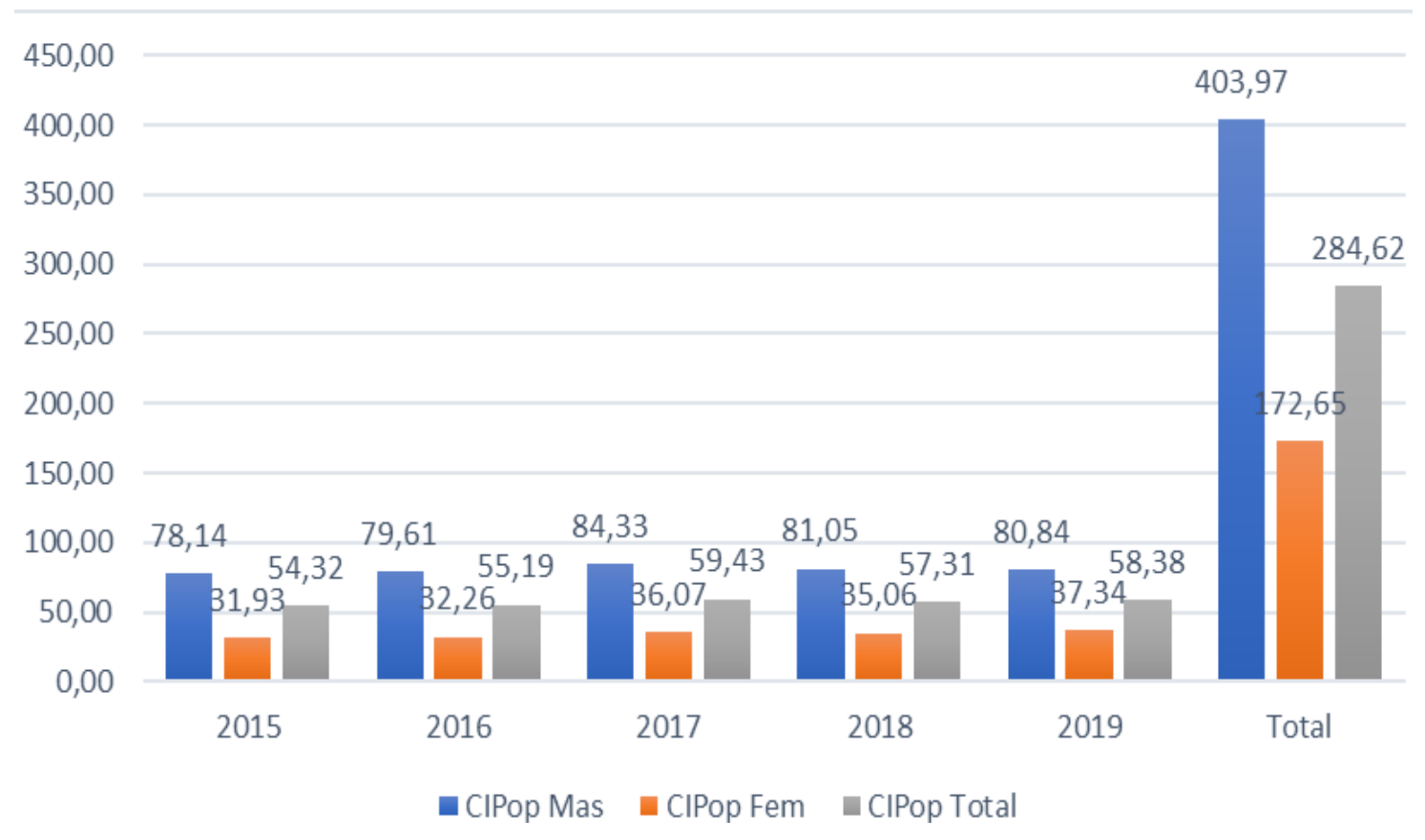

Fonte: Ministério da Saúde - Sistema de Informações Hospitalares do SUS (SIH/SUS).

Observa-se na Tabela 3, que a causa do óbito mais prevalente em todos os grupos de faixa etária foi exposição a outros fatores específicos, afetando com maior incidência a faixa etária que compreende os adultos de 20 a 59 anos $(n=65.155)$, visto que, esta causa foi responsável por 56,75\% do total de óbitos nessa faixa etária. 
Tabela 3 - Proporção de óbitos por causas externas segundo agrupamento de faixa etária e categoria das causas. Pernambuco, 2015 a 2019.

\begin{tabular}{|c|c|c|c|c|c|c|c|c|}
\hline \multirow{2}{*}{ Faixa etária } & \multicolumn{2}{|c|}{1 a 19 anos } & \multicolumn{2}{|c|}{20 a 59 anos } & \multicolumn{2}{|c|}{60 anos e mais } & \multicolumn{2}{|c|}{ Total } \\
\hline & $\mathbf{N}^{\mathbf{0}}$ & $\%$ & $\mathbf{N}^{\mathbf{0}}$ & $\%$ & $\mathbf{N}^{\mathbf{0}}$ & $\%$ & $\mathbf{N}^{\mathbf{0}}$ & $\%$ \\
\hline Pedestre traum colis veic a pedal & 1515 & 31,54 & 2662 & 55,42 & 626 & 13,03 & 4803 & 2,30 \\
\hline Pedestre traum outr acid transp e NE & 275 & 19,22 & 883 & 61,71 & 273 & 19,08 & 1431 & 0,69 \\
\hline Motociclista traum outr acid transp e NE & 1776 & 11,93 & 12501 & 83,98 & 608 & 4,08 & 14885 & 7,14 \\
\hline Outr quedas no mesmo nivel & 2233 & 8,33 & 13588 & 50,70 & 10981 & 40,97 & 26802 & 12,86 \\
\hline Queda s/especificacao & 568 & 15,93 & 2333 & 65,44 & 664 & 18,63 & 3565 & 1,71 \\
\hline Exposicao a outr fatores espec & 30320 & 26,41 & 65155 & 56,75 & 19342 & 16,85 & 114817 & 55,07 \\
\hline Exposição a fatores NE & 3977 & 19,90 & 12991 & 65,01 & 3014 & 15,08 & 19982 & 9,58 \\
\hline Agressao objeto cortante ou penetrante & 272 & 14,45 & 1551 & 82,37 & 60 & 3,19 & 1883 & 0,90 \\
\hline Outr fatos ou eventos espec intenc $\mathrm{n}$ det & 1483 & 15,36 & 5586 & 57,84 & 2588 & 26,80 & 9657 & 4,63 \\
\hline Sequelas de outr causas externas & 1451 & 18,22 & 4404 & 55,31 & 2108 & 26,47 & 7963 & 3,82 \\
\hline Circunst relat condicoes do modo de vida & 742 & 27,55 & 1111 & 41,26 & 840 & 31,19 & 2693 & 1,29 \\
\hline Total & 44612 & 100 & 122765 & 100 & 41104 & 100 & 208481 & 100 \\
\hline
\end{tabular}

Fonte: Ministério da Saúde - Sistema de Informações Hospitalares do SUS (SIH/SUS).

Levando em consideração os dados apresentados anteriormente, pode-se afirmar que o estudo em questão pode apresentar algumas restrições no momento de análise dos seus resultados, esse aspecto se explica pelo fato de que não são todas as mortes, que anteriormente ao ocorrido, os indivíduos tenham dado entrada em uma unidade hospitalar ou tenham tido qualquer tipo de atendimento, além de ser ressaltado a questão de que alguns são atendidos por estabelecimentos de saúde particulares que não fazem o lançamento dos dados no SIH/SUS (Machado, Martins, Leite, 2016; Melione, Jorge, 2008; Filho, Bermudez, Hamann, 2021).

Sendo que para ser registrada tal morte por causa externa no Sistema de Informações Hospitalares é importante que ocorra o registro do paciente no sistema, por meio do formulário virtual de Autorização de Internação Hospitalar (AIH) (Machado, Martins, Leite, 2016; Melione, Jorge, 2008).

Além do mais, algumas vítimas após sofrer alguma lesão são rapidamente liberadas do serviço de emergência e os sistemas de informações hospitalares são bastante limitados em relação a disponibilidade de informações clínicas, contribuindo para que não sejam retratados dos os óbitos ocorridos devido a causas externas (Machado, Martins, Leite, 2016; Melione, Jorge, 2008).

No estudo presente é perceptível a predominância de óbitos no sexo masculino, se comparado a população feminina. Os fatores que influenciam diretamente são inúmeros, tais como: Raça/cor, Estado Civil, Escolaridade, entre outros (Oliveira, Correa, Silva, Mozer, Medeiros, 2017; Silva, Alvarenga, Silva, Barbosa, Rocha, 2010; Fuck, Assis, Tornquist, 2020).

As circunstâncias mais observadas nas morte para esta população, de acordo com a literatura, estão relacionadas aos homicídios e acidentes de trânsito, aspectos culturais também se fazem presente, tais como os fatores socioeconômicos, uma vez que, os homens costumam procurar os serviços de saúde bem menos que as mulheres, como também não detém conhecimento sobre ações preventivas e profiláticas que de forma consequente podem ser prejudiciais (Oliveira, Correa, Silva, 
Mozer, Medeiros, 2017; Silva, Alvarenga, Silva, Barbosa, Rocha, 2010; Silva, Lima, Barbosa, Lima, Bandeira, Santos, Silva, Simoneti, 2021).

A maior taxa de mortalidade, ocasionada por causas externas, na faixa etária de 20 a 59, também vai de encontro com outras pesquisas; de tal maneira que esse fato pode ser explicado devido principalmente às desigualdades sociais e falta de estabilidade que cercam os jovens, primordialmente relacionado ao que diz respeito a violência e acidentes de transporte, evidenciando-se tais aspectos pelos dados ofertados no presente estudo (Neves, Garcia, 2015; Melo, 2014; Nadanovsky, Santos, 2021).

\section{Conclusão}

É imprescindível analisar o índice de mortalidade por causas externas através do SIH, uma vez que ele fornece os dados para o desenvolvimento de estudos como este, que se caracteriza como sendo de suma importância. Porém, vale ressaltar apenas a necessidade de uma atualização no referido sistema, a fim de torná-lo mais efetivo em alguns casos, como por exemplo, melhorando as notificações na rede privada de atendimento hospitalar, o que levaria a um número mais fidedigno na amostra coletada e evitaria também um aumento das subnotificações.

Além do mais, espera-se ainda que todas as evidências levantadas neste estudo sejam influenciadoras e incentivadoras do aprimoramento das políticas públicas de saúde já existentes e colabore com o desenvolvimento de novas políticas voltadas para a prevenção e consequente diminuição das taxas de mortalidade provocadas por causas externas que podem e devem ser evitadas e preveníveis.

Assim, a presente pesquisa se apresenta como incentivo para o desenvolvimento de outras pesquisas com metodologias diferentes que venham a abordar mais a fundo a temática trabalhada em questão, explanando os principais fatores que levam a um número elevado de mortes por tais causas, como também as intervenções que podem ser aplicadas em tempo oportuno que possam contribuir para sua diminuição.

\section{Referências}

Arruda, S. G., \& Rodrigues, E. K. (2020). Mortalidade por causas externas na região norte do espírito santo, 2010 a 2018. Revista Artigos, 20 , 4258.

Assig, O., Fuckj, A. B., \& Tornquistc, S. (2020). Análise da mortalidade por causas externas, Santa Catarina, 2008 a 2017. Revista Eletrônica Acervo Saúde, 12(8), 3381.

Assis, G. O., Fuck, J. A. B., \& Tornquist, C. S. (2020). Análise da mortalidade por causas externas, Santa Catarina, 2008 a 2017. Revista Eletrônica Acervo Saúde, 12 (8), e3381-e3381.

Batista, J., Barreto, M. S., Merino, M. F. G., Fracasso, N. V., \& Baldissera, V. D. A. (2018). Perfil epidemiológico da mortalidade por causas externas entre beneficiários de planos de saúde no Brasil. Revista de Enfermagem do Centro-Oeste Mineiro, 8.

Bermudez, X. P., Filho, A. M., \& Hamann, E. M. (2021). Frequência e fatores associados ao registro inespecífico de óbitos por causas externas no Brasil: estudo transversal, 2017. Epidemiol. Serv. Saúde, 30 (2), 2020-452.

Boone, D. L., Costa, T. M. T., Tana, D. B., Lopes, S. C., Cortes, M. C. S., Freitas, E. D., \& Alves, W. A. (2018). Serviço de Atendimento Móvel de Urgência: perfil dos atendimentos por causas externas. Rev Soc Bras Clin Med., 16 (3), 134-9.

Correa, A. C. P., Mozer, I. T., Medeiros, R. M. K., Oliveira, J. C. A. X., \& Silva, L. A. (2017). Perfil Epidemiológico da Mortalidade Masculina: Contribuições para Enfermagem. Cogitare Enferm, 2 (22), 49724.

Cruz, M. C. (2017). Análise epidemiológica da mortalidade de adultos e idosos da II Região de Saúde de Pernambuco. Vitória de Santo Antão, $26,258$.

Donalísio, M. R., Melo, M. C., \& Pimenta, A. M. (2016). Perfil epidemiológico de idosos com aids na macrorregião de saúde de Belo Horizonte. Revista de Enfermagem do Centro Oeste Mineiro, 1 (6), 2020-2033.

Fuck, J. A. B., Assis, G. O.; \& Tornquist, C. S. (2020). Análise da mortalidade por causas externas, Santa Catarina, 2008 a 2017. Revista Eletrônica Acervo Saúde, 12(8), e3381.

Garcia, L. P., \& Neves, A. C. M. (2015). Mortalidade de jovens brasileiros: perfil e tendências no período 2000- 2012. Epidemiol. Serv. Saúde, Brasília, v.24 (4), 595-606. 
Research, Society and Development, v. 10, n. 12, e69101220121, 2021

(CC BY 4.0) | ISSN 2525-3409 | DOI: http://dx.doi.org/10.33448/rsd-v10i12.20121

IBGE. Instituto Brasileiro de Geografia Estatística. Cidades e Estados. 〈https://www.ibge.gov.br/cidades-e-estados/pe.html>,

Leite, I. C., Machado, J. P., \& Martins, M. (2016). Qualidade das bases de dados hospitalares no Brasil: Alguns Elementos. Revista Brasileira de Epidemiologia, 19, 567-581.

Lignani, L. O., \& Villela, L. C. M. (2013). Estudo descritivo sobre a morbidade hospitalar por causas externas em Belo Horizonte, Estado de Minas Gerais, Brasil, 2008-2010. Epidemiol. Serv. Saúde, Brasília, 22 (2), 225-234.

Messias, K. L. M. (2016). Qualidade da informação dos óbitos por causas externas em Fortaleza, Ceará, Brasil. Ciência \& Saúde Coletiva, 21 (4), $1255-1266$.

Messias, M. M., Bandeira, J. R., Lopes, A. B., Silva, L. L. D., \& Curado, P. F. (2018). Mortalidade por causas externas: revisão dos dados do Sistema de Informação de Mortalidade. Rev Soc Bras Clin Med., 16 (4), 218-21.

Melo, M. C. (2014). Perfil da mortalidade hospitalar por causas externas: Óptica sobre a notificação dos dados. Rev enferm UFPE on line, 8 (7), $1898-903$.

Nadanovsky, P., \& Santos, A. P (2021). Saúde Amanhã: textos para discussão: mortes por causas externas no Brasil: previsões para as próximas duas décadas. Fundação Oswaldo Cruz,60 (56), 56-58.

Oliveira, J. C. A., Correa, A. C. P., Silva, L. A., Mozer, I. T., \& Medeiros, R. M. K. (2017). Perfil Epidemiológico da mortalidade masculina: Contribuições para enfermagem. Cogitare Enferm., 22 (2), e49724.

Silva, S. K. A., Lima, B. L., Barbosa, D. A. M., Lima, M. A. M., Bandeira, T. D., Santos, I. H. O. L., Silva, A. S. R., \& Simoneti, R. A. A. O. (2021). Óbitos por causas externas no Brasil: um estudo ecológico temporal de 2014 a 2018. Brazilian Journal of Development, 7 (7), 67049-67059.

Silva, M. E. D. C., Alvarenga, W. A., Silva, S. S., Barbosa, L. D. C. S., \& Rocha, S. S. (2010). Resistência do homem às ações de saúde: percepção de enfermeiras da estratégia saúde da família. Revista Interdisciplinar NOVAFAPI, 3(3), 21-25. 